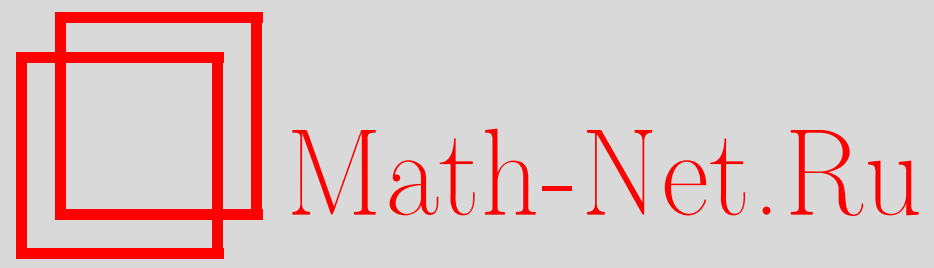

В. А. Федченко, Показатели рассеивания линейной среды AES-подобных алгоритмов шифрования, Матем. вопр. криптогр., 2017, том 8, выпуск 3, 109-126

DOI: https://doi.org/10.4213/mvk234

Использование Общероссийского математического портала Math-Net.Ru подразумевает, что вы прочитали и согласны с пользовательским соглашением

http: //www.mathnet.ru/rus/agreement

Параметры загрузки:

IP : 54.224 .187 .69

26 апреля 2023 г., $17: 21: 26$ 
МАТЕМАТИЧЕСКИЕ ВОПРОСЫ КРИПТОГРАФИИ

2017 T. 8 № 3 C. 109-126

УДК 519.719.2+519.712

\title{
Показатели рассеивания линейной среды AES-подобных алгоритмов шифрования
}

\author{
В. А. Федченко
}

\author{
Белорусский государственный университет, Минск
}

Получено 10.05.2017

\begin{abstract}
Аннотация. В качестве модели для AES-подобных алгоритмов шифрования рассматриваются XSLP-шифры с условием, что все перестановки $P$ являются равномерно рассеивающими, а блоки матриц преобразований $L$ имеют фиксированный линейный (разностный) коэффициент рассеивания $\rho$. При всех допустимых $\rho$ получена точная нижняя оценка минимального количества локальных подстановок, участвующих в системах линейных (разностных) вероятностных соотношений для произвольного числа итераций XSLP-шифра.
\end{abstract}

Ключевые слова: AES, AES-подобные алгоритмы, XSLP-шифры, линейный криптоанализ, разностный криптоанализ, активные S-блоки

\section{Diffusion rates of linear medium in AES-like ciphers}

\section{A. Fedchenko}

Belarusian State University, Minsk

\begin{abstract}
We model AES-like algorithms by XSLP-ciphers having uniformly diffusive permutations $P$ and fixed linear (differential) branch number $\rho$ of blocks of transformation matrices $L$. For all admissible values of $\rho$ and arbitrary number of rounds the exact lower bound for the number of active S-boxes in systems of linear (differential) probabilistic relations is obtained.
\end{abstract}

Keywords: AES, AES-like algorithms, XSLP-ciphers, linear cryptanalysis, differential cryptanalysis, active S-boxes 


\section{XSLP-шифры в качестве модели для AES-подобных алгоритмов шифрования}

Алгоритм шифрования AES [8] в настоящее время является де-факто мировым стандартом шифрования. Помимо практической стойкости и эффективности в эксплуатации достоинством этого алгоритма является доказуемая стойкость к разностному и линейному методам криптографического анализа. Это свойство во многом обеспечивается продуманной структурой алгоритма AES.

Структура алгоритма AES характерна для многих других алгоритмов шифрования, неформально называемых «AES-подобными». В качестве примеров можно привести алгоритмы Anubis [3], BKSQ [7], Crypton [10], GrandCru [5], Square [6] и другие. Общность структуры AES-подобных алгоритмов шифрования позволяет проводить их исследование с единых позиций.

В качестве модели для исследования AES-подобных алгоритмов шифрования целесообразно использовать определение XSLP-схемы [2]. Положим, что длина блока равна $n=m k^{2}$ битов. Алгоритм, реализующий XSLP-схему, оперирует с $m k^{2}$-векторами $x \in V_{m k^{2}}$, состоящими из $m k$-векторов в количестве $k$ штук:

$$
x=(\underbrace{x_{0}, x_{1}, \ldots, x_{k-1}}_{m k \text {-вектор } 0}, \underbrace{x_{k}, x_{k+1}, \ldots, x_{2 k-1}}_{m k \text {-вектор } 1}, \ldots, \underbrace{x_{(k-1) k}, x_{(k-1) k+1}, \ldots, x_{k^{2}-1}}_{m k \text {-вектор } k-1}),
$$

где $x_{j} \in V_{m}=\mathrm{GF}(2)^{m}\left(j=0,1, \ldots, k^{2}-1\right)-m$-векторы. Для $m$-векторов удобно использовать нумерацию, связанную с номерами $m k$-векторов, в которые они входят. Пусть $j$ - номер $m$-вектора. Определим его альтернативный номер как пару чисел $\left(j_{0}, j_{1}\right), j_{0}, j_{1}=0,1, \ldots, k-1$, где $j=j_{0}+k j_{1}$, $j_{0}$ - младшая цифра, $j_{1}-$ старшая. При такой записи $j_{0}$ - номер $m$-вектора в $m k$-векторе с номером $j_{1}$. В этом случае исходный $m k^{2}$-вектор $x \in V_{m k^{2}}$ имеет вид

$$
x=(\underbrace{x_{(0,0)}, \ldots, x_{(0, k-1)}}_{m k \text {-вектор } 0}, \underbrace{x_{(0,1)}, \ldots, x_{(k-1,1)}}_{m k \text {-вектор } 1}, \ldots, \underbrace{x_{(0, k-1)}, \ldots, x_{(k-1, k-1)}}_{m k \text {-вектор } k-1}),
$$

где $x_{\left(j_{0}, j_{1}\right)} \in V_{m}, j_{0}, j_{1}=0,1, \ldots, k-1$. 
Базовый блочный алгоритм зашифрования XSLP-схемы состоит из $r$ однотипных итераций $R_{i}: V_{m k^{2}} \rightarrow V_{m k^{2}}, \quad i=1,2, \ldots, r$. Каждая итерация представляет собой произведение биективных преобразований $X_{i}, S_{i}, L_{i}, \widehat{P}_{i}$, действующих на $m k^{2}$-векторах:

$$
\begin{aligned}
& R_{i}=X_{i} \cdot S_{i} \cdot L_{i} \cdot \widehat{P}_{i}, \quad b=R_{i}(a), \quad b=\widehat{P}_{i}\left(L_{i}\left(S_{i}\left(X_{i}(a)\right)\right)\right), \\
& a \stackrel{X_{i}}{\longmapsto} u \stackrel{S_{i}}{\longmapsto} v \stackrel{L_{i}}{\longmapsto} w \stackrel{\widehat{P}_{i}}{\longmapsto} b, \quad a, b, u, v, w \in V_{m k^{2}} .
\end{aligned}
$$

Преобразование $X_{i}: V_{m k^{2}} \rightarrow V_{m k^{2}}, i=1,2, \ldots, r$, представляет собой наложение итерационного ключа $z_{i} \in V_{m k^{2}}$ побитово по модулю 2 :

$$
\begin{aligned}
u=X_{i}(a) & =a \oplus z_{i}=\left(\left(a_{0} \oplus z_{i, 0}\right),\left(a_{1} \oplus z_{i, 1}\right), \ldots,\left(a_{k^{2}-1} \oplus z_{i, k^{2}-1}\right)\right)= \\
& =\left(\left(a_{0,0} \oplus z_{i,(0,0)}\right),\left(a_{1,0} \oplus z_{i,(1,0)}\right), \ldots,\left(a_{k-1, k-1} \oplus z_{i,(k-1, k-1)}\right)\right) .
\end{aligned}
$$

Преобразование $S_{i}: V_{m k^{2}} \rightarrow V_{m k^{2}}, \quad i=1,2, \ldots, r$, представляет собой замену $m$-векторов под действием локальных подстановок $\pi_{i, j} \in S\left(V_{m}\right)$, $j=0,1, \ldots, k^{2}-1$ :

$$
\begin{aligned}
v=S_{i}(u) & =\left(\pi_{i, 0}\left(u_{0}\right), \pi_{i, 1}\left(u_{1}\right), \ldots, \pi_{i, k^{2}-1}\left(u_{k^{2}-1}\right)\right)= \\
& =\left(\pi_{i,(0,0)}\left(u_{0,0}\right), \pi_{i,(1,0)}\left(u_{1,0}\right), \ldots, \pi_{i,(k-1, k-1)}\left(u_{k-1, k-1}\right)\right) .
\end{aligned}
$$

Преобразование $L_{i}: V_{m k^{2}} \rightarrow V_{m k^{2}}, i=1,2, \ldots, r$, представляет собой умножение $m k$-векторов справа на невырожденные матрицы $B_{i, j_{1}} \in \mathrm{GL}(m k, 2)$, $j_{1}=0,1, \ldots, k-1$ :

$$
\begin{aligned}
w=L_{i}(v)=( & \left(v_{0,0}, v_{1,0}, \ldots, v_{k-1,0}\right) \cdot B_{i, 0},\left(v_{0,1}, v_{1,1}, \ldots, v_{k-1,1}\right) \cdot B_{i, 1}, \ldots \\
& \left.\ldots,\left(v_{0, k-1}, v_{1, k-1}, \ldots, v_{k-1, k-1}\right) \cdot B_{i, k-1}\right) .
\end{aligned}
$$

Преобразование $\widehat{P}_{i}: V_{m k^{2}} \rightarrow V_{m k^{2}}, i=1,2, \ldots, r$, представляет собой перестановку $m$-векторов по правилу

$$
P_{i}=\left(\begin{array}{cccc}
0 & 1 & \cdots & k^{2}-1 \\
P_{i}(0) & P_{i}(1) & \cdots & P_{i}\left(k^{2}-1\right)
\end{array}\right)=\left(\begin{array}{cccc}
P_{i}^{-1}(0) & P_{i}^{-1}(1) & \cdots & P_{i}^{-1}\left(k^{2}-1\right) \\
0 & 1 & \cdots & k^{2}-1
\end{array}\right),
$$

где $P_{i} \in S\left(\overline{0, k^{2}-1}\right)$, и предполагается, что $m$-вектор с номером 0 переходит на место $P_{i}(0), m$-вектор с номером 1 переходит на место $P_{i}(1)$ и так далее:

$$
b=\widehat{P}_{i}(w)=\left(w_{P_{i}^{-1}(0)}, w_{P_{i}^{-1}(1)}, \ldots, w_{P_{i}^{-1}\left(k^{2}-1\right)}\right) .
$$


Функциональная схема одной итерации XSLP-схемы изображена на рис. 1.

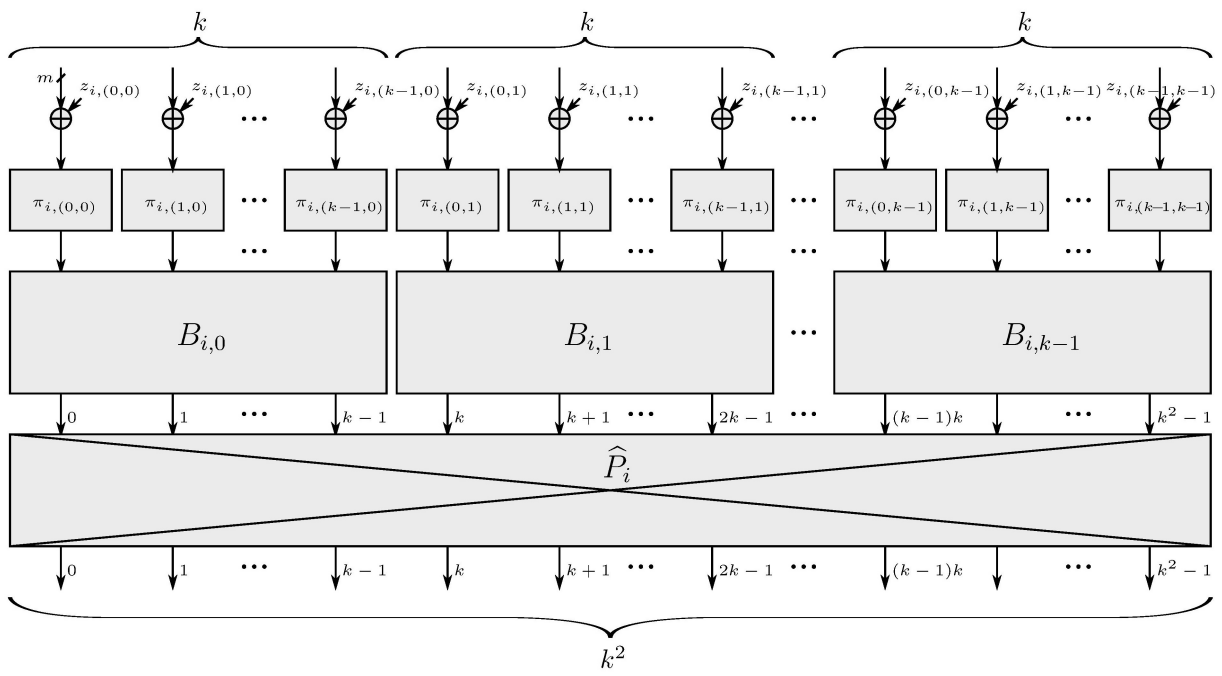

Рис. 1. Функциональная схема $i$-й итерации XSLP-схемы

Замечание 1. В сущности, на рис. 1 представлены также XSL-схемы и $\mathrm{SP}$-сети. С одной стороны, исключив преобразование $\widehat{P}_{i}$, получим $k$ подстановок, заданных одной итерацией классических XSL-схем с основным алфавитом GF(2). С другой стороны, объявив эти XSL-схемы отдельными подстановками на $V_{m k}$, получим классическую SP-сеть с основным алфавитом $V_{m}$. Также XSL-схему можно получить, рассматривая преобразования $L_{i}$ и $\widehat{P}_{i}$ как единое линейное преобразование. Линейное преобразование XSLP-схем, заданное в виде суперпозиции преобразований $\widehat{P}_{i}$ и $L_{i}$, где последнее состоит из $B_{i, j_{1}}, j=0, \ldots, k-1$, является удачным компромиссом между перестановками $m k^{2}$ битов (как в классических SP-сетях) и максимально рассеивающими матрицами из $\operatorname{GL}\left(m k^{2}, 2\right)$ (как в XSL-схемах, см. (10) и (11) ниже). Как правило, классические SP-сети уступают XSLP-схемам с точки зрения аналитической сложности, а максимально рассеивающие матрицы XSL-схем сложнее для реализации.

Основными ключевыми параметрами XSLP-схемы являются итерационные ключи $z_{i}, \quad i=1,2, \ldots, r$, представляющие собой $m k^{2}$-векторы. Алгоритм развертывания итерационных ключей не специфицируется. Дополнительными ключевыми параметрами XSLP-схемы могут являться элементы итерационных преобразований (локальные подстановки, матрицы, перестановки $m$-векторов). 
Таким образом определено понятие XSLP-схемы. В [2] предлагается также более общее понятие XSLP-шифра, структура итерации которого подходит под определение XSLP-схемы при любом порядке следования преобразований $X_{i}, S_{i}, L_{i}, \widehat{P}_{i}$. Например, алгоритм AES относится к XSLP-шифрам и построен по XSLP-схеме при $m=8, k=4$.

\section{2.Линейный и разностный методы криптографического анализа XSLP-шифров}

Криптографический анализ XSLP-шифров линейным и разностным методами можно проводить по единой схеме, используя их двойственность [1]. Напомним эту схему на примере линейного метода в соответствии с изложенным в $[1,2]$.

Пусть $F: V_{m k^{2}} \rightarrow V_{m k^{2}}$ - некоторое биективное преобразование, действующее на векторах-строках длины $m k^{2}$. Пара векторов-столбцов $p^{\prime}, h^{\prime \prime} \in V_{m k^{2}}^{*} \backslash\{0\}$, где $V_{m k^{2}}^{*}-$ пространство векторов-стобцов, задает линейное вероятностное соотношение для $F$, как бы приближенное равенство, записываемое в виде $a \cdot p^{\prime} \simeq b \cdot h^{\prime \prime}$, где $a, b \in V_{m k^{2}}, b=F(a), a$ выбирается из $V_{m k^{2}}$ случайно и равновероятно. Соответствующее точное равенство выполняется с некоторой вероятностью:

$$
\mathrm{P}\left\{a \cdot p^{\prime}=b \cdot h^{\prime \prime}\right\}=\frac{1+\delta_{p^{\prime}, h^{\prime \prime}}}{2},
$$

где $\delta_{p^{\prime}, h^{\prime \prime}} \in[-1,1]$ - преобладание линейного вероятностного соотношения. Чем больше $\delta_{p^{\prime}, h^{\prime \prime}}$, тем более оправдан термин «приближенное равенство».

Основной задачей линейного метода анализа шифрпреобразований является нахождение линейного вероятностного соотношения, которое выполняется с большим модулем преобладания при любом ключе. Эта задача обычно решается путем нахождения так называемой системы согласованных соотномений для составных частей шифрпреобразования.

Пусть $F: V_{m k^{2}} \rightarrow V_{m k^{2}}$ - произведение $t$ биективных преобразований, действующих на векторах-строках длины $m k^{2}: F=F_{1} \cdot F_{2} \cdot \ldots \cdot F_{t}$. Пусть для каждого $j \in \overline{1, t}$ векторы-столбцы $p_{j}^{\prime}, h_{j}^{\prime \prime} \in V_{m k^{2}}^{*} \backslash\{0\}$ задают линейное вероятностное соотношение для преобразования $F_{j}$, выполняющееся с преобладанием $\delta_{p_{j}^{\prime}, h_{j}^{\prime \prime}}$ Условие согласованности в данном случае означает, что $h_{j}^{\prime \prime}=p_{j+1}^{\prime}$ для всех $j \in \overline{1, t-1}$. Тогда $p^{\prime}=p_{1}^{\prime}$ и $h^{\prime \prime}=h_{t}^{\prime \prime}$ задают линейное вероятностное соотношение для преобразования $F$. В качестве оценки модуля преобладания $\left|\delta_{p^{\prime}, h^{\prime \prime}}\right|$ этого соотношения используют величину $\widetilde{\delta}_{p^{\prime}, h^{\prime \prime}}=\prod_{j=1}^{t}\left|\delta_{p_{j}^{\prime}, h_{j}^{\prime \prime}}\right|$ и решают задачу по ее максимизации. 
B XSLP-шифрах в качестве отдельных преобразований, произведение которых составляет все шифрпреобразование $E=R_{1} \cdot R_{2} \cdot \ldots \cdot R_{r}$, выступают $X_{i}, \quad S_{i}, \quad L_{i}, \widehat{P}_{i}, \quad R_{i}=X_{i} \cdot S_{i} \cdot L_{i} \cdot \widehat{P}_{i}, \quad i=1,2, \ldots, r$. Рассмотрим свойства их линейных вероятностных соотношений.

Для преобразования $S_{i}$ обозначим векторы-столбцы, задающие линейное вероятностное соотношение, через $l_{i}^{\prime}, l_{i}^{\prime \prime} \in V_{m k^{2}}^{*} \backslash\{0\}$. Представим $l_{i}^{\prime}$ и $l_{i}^{\prime \prime}$ в виде $l_{i}^{\prime}=\left(\left(l_{i, 0}^{\prime}\right)^{\top},\left(l_{i, 1}^{\prime}\right)^{\top}, \ldots,\left(l_{i, k^{2}-1}^{\prime}\right)^{\top}\right)^{\top}, l_{i}^{\prime \prime}=\left(\left(l_{i, 0}^{\prime \prime}\right)^{\top},\left(l_{i, 1}^{\prime \prime}\right)^{\top}, \ldots\right.$ $\left.\ldots,\left(l_{i, k^{2}-1}^{\prime \prime}\right)^{\top}\right)^{\top}$, где $l_{i, j}^{\prime}, l_{i, j}^{\prime \prime} \in V_{m}^{*}, \quad j=0,1, \ldots, k^{2}-1, \quad \top-$ знак транспонирования. Очевидно, что

$$
\left|\delta_{l_{i}^{\prime}, l_{i}^{\prime \prime}}\right|=\prod_{j=0}^{k^{2}-1}\left|\delta_{l_{i, j}^{\prime}, l_{i, j}^{\prime \prime}}\right|,
$$

где $l_{i, j}^{\prime}, l_{i, j}^{\prime \prime}$ задают линейные вероятностные соотношения с преобладаниями $\delta_{l_{i, j}^{\prime}, l_{i, j}^{\prime \prime}}$ для локальных подстановок $\pi_{i, j}$.

Для преобразований $X_{i}, \quad L_{i}, \quad \widehat{P}_{i}$ обозначим векторы-столбцы, задающие линейные вероятностные соотношения, соответственно $p_{i}^{\prime}, p_{i}^{\prime \prime} \in V_{m k^{2}}^{*}$, $t_{i}^{\prime}, t_{i}^{\prime \prime} \in V_{m k^{2}}^{*}$ и $h_{i}^{\prime}, h_{i}^{\prime \prime} \in V_{m k^{2}}^{*}$. Покажем, что они выражаются через $l_{i}^{\prime}, l_{i}^{\prime \prime}$ благодаря согласованности, предполагающей равенства $p_{i}^{\prime \prime}=l_{i}^{\prime}, l_{i}^{\prime \prime}=t_{i}^{\prime}$ и $t_{i}^{\prime \prime}=h_{i}^{\prime}$, а также $h_{i}^{\prime \prime}=p_{i+1}^{\prime}, \quad i=1,2, \ldots, r-1$.

1. Рассмотрим преобразование $X_{i}$. Можно заметить, что вне зависимости от итерационного ключа $z_{i}$ справедливо соотношение

$$
\left|\delta_{p_{i}^{\prime}, p_{i}^{\prime \prime}}\right|= \begin{cases}1, & \text { если } p_{i}^{\prime}=p_{i}^{\prime \prime}, \\ 0 & \text { в противном случае. }\end{cases}
$$

Исключая нуль и используя согласованность, имеем $p_{i}^{\prime}=p_{i}^{\prime \prime}=l_{i}^{\prime}$.

2. Рассмотрим преобразование $L_{i}$. Оно задается умножением на матрицу, также обозначаемую $L_{i}$. Тогда

$$
\left|\delta_{t_{i}^{\prime}, t_{i}^{\prime \prime}}\right|= \begin{cases}1, & \text { если } t_{i}^{\prime}=L_{i} \cdot t_{i}^{\prime \prime}, \\ 0 & \text { в противном случае. }\end{cases}
$$

Исключая нуль и используя согласованность, имеем $t_{i}^{\prime}=l_{i}^{\prime \prime}, t_{i}^{\prime \prime}=L_{i}^{-1} \cdot t_{i}^{\prime}=$ $=L_{i}^{-1} \cdot l_{i}^{\prime \prime}$.

3. Рассмотрим преобразование $\widehat{P}_{i}$. С учетом равенств $h_{i}^{\prime}=t_{i}^{\prime \prime}=L_{i}^{-1} \cdot l_{i}^{\prime \prime}$ аналогично предыдущему имеем: $h_{i}^{\prime \prime}=\widehat{P}_{i}^{-1} \cdot h_{i}^{\prime}=\widehat{P}_{i}^{-1} \cdot L_{i}^{-1} \cdot l_{i}^{\prime \prime}=$ $\left(L_{i} \cdot \widehat{P}_{i}\right)^{-1} \cdot l_{i}^{\prime \prime}$. Заметим, что если $h_{i}^{\prime}, h_{i}^{\prime \prime} \in V_{m k}^{*}$ составлены соответственно из векторов-столбцов $h_{i, j}^{\prime}, h_{i, j}^{\prime \prime} \in V_{m}^{*}$, то $h_{i, j}^{\prime \prime}=h_{i, P^{-1}(j)}^{\prime}$, $j=0,1, \ldots, k^{2}-1$. 
Поскольку каждое из преобразований $X_{i}, S_{i}, \widehat{P}_{i}$ преобразует отдельные $m$-векторы независимо друг от друга, в соотношениях для них любым $p_{i, j^{\prime}}^{\prime}, l_{i, j^{\prime}}^{\prime}, h_{i, j^{\prime}}^{\prime} \in V_{m}^{*} \backslash\{0\}, \quad j^{\prime}=0,1, \ldots, k^{2}-1$, соответствуют вполне определенные $p_{i, j^{\prime \prime}}^{\prime \prime}, l_{i, j^{\prime \prime}}^{\prime \prime}, h_{i, j^{\prime \prime}}^{\prime \prime} \in V_{m}^{*} \backslash\{0\}, j^{\prime \prime}=0,1, \ldots, k^{2}-1$. Причем $j^{\prime}=j^{\prime \prime}$ в случае преобразований $X_{i}, S_{i}$, а $j^{\prime \prime}=P_{i}\left(j^{\prime}\right)$ в случае преобразования $\widehat{P}_{i}$.

Линейное вероятностное соотношение для $R_{i}=X_{i} \cdot S_{i} \cdot L_{i} \cdot \widehat{P}_{i}$ задается векторами-столбцами $l_{i}^{\prime}, h_{i}^{\prime \prime}, \quad i=1,2, \ldots, r$, причем (по согласованности) $h_{i}^{\prime \prime}=l_{i+1}^{\prime}, \quad i=1,2, \ldots, r-1$. Линейное вероятностное соотношение для $E=R_{1} \cdot R_{r} \cdot \ldots \cdot R_{r}$ задается векторами-столбцами $l_{1}^{\prime}, h_{r}^{\prime \prime}$.

Взаимосвязь между линейными вероятностными соотношениями для составных частей XSLP-схемы представлена на рис. 2.

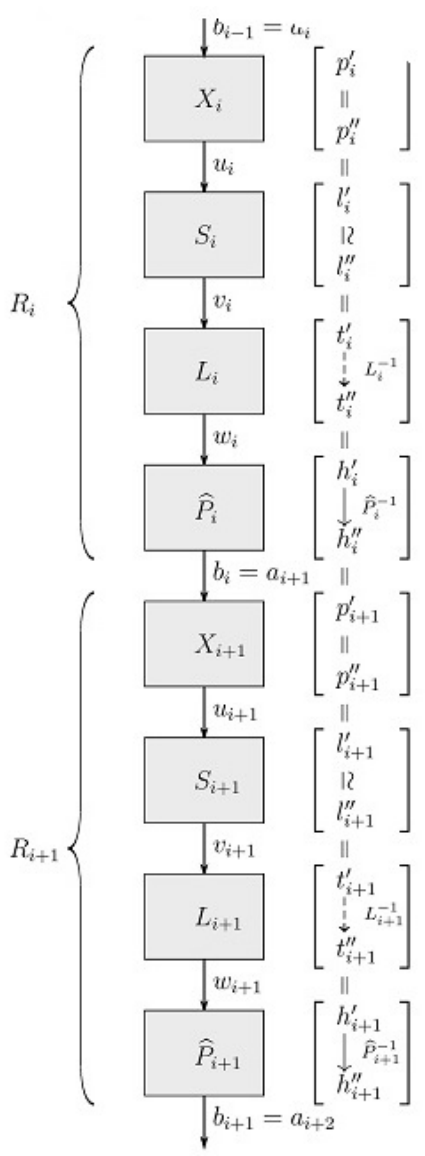

Рис. 2. Иллюстрация к линейным вероятностным соотношениям для XSLP-схемы 
Принимая во внимание указанные выше свойства, можно заметить, что для $r$ итераций XSLP-шифра оценка модуля преобладания выражается формулой

$$
\widetilde{\delta}_{\mathcal{L}}(r)=\prod_{i=1}^{r} \prod_{j=0}^{k^{2}-1}\left|\delta_{l_{i, j}^{\prime}, l_{i, j}^{\prime \prime}}\right|,
$$

где $\mathcal{L}=\left\{\left(l_{i, j}^{\prime}, l_{i, j}^{\prime \prime}\right), i=1,2, \ldots, r, j=0,1, \ldots, k^{2}-1\right\}$ - согласованная система линейных вероятностных соотношений для локальных подстановок $\pi_{i, j}$. При этом можно считать, что для всех $i \in \overline{1, r}, j \in \overline{0, k^{2}-1}$ либо $l_{i, j}^{\prime}=0, l_{i, j}^{\prime \prime}=0$, либо $l_{i, j}^{\prime} \neq 0, l_{i, j}^{\prime \prime} \neq 0$. Другие две ситуации в случае подстановок заведомо приводят к нулевому преобладанию $\delta_{l_{i, j}^{\prime}, l_{i, j}^{\prime \prime}}$.

Пусть $W$ - все возможные системы $\mathcal{L}$, удовлетворяющие условиям $l_{i, j}^{\prime} \neq 0$ и $l_{i, j}^{\prime \prime} \neq 0$ при некоторых $i \in \overline{1, r}$ и $j \in \overline{0, k^{2}-1}$. Тогда оценка стойкости XSLP-шифра к линейному методу предполагает нахождение точного значения или оценки сверху величины

$$
\widetilde{\delta}_{\max }(r)=\max _{\mathcal{L} \in W} \widetilde{\delta}_{\mathcal{L}}(r)
$$

Будем считать, что для локальных подстановок в рассматриваемых шифрах не существует ненулевых соотношений, выполняющихся с модулем преобладания 1. Тогда важное значение имеет количество сомножителей в (5). Обозначим через

$$
\theta_{\mathcal{L}}(r)=\left|\left\{i=1,2, \ldots, r, j=0,1, \ldots, k^{2}-1: l_{i, j}^{\prime} \neq 0, l_{i, j}^{\prime \prime} \neq 0\right\}\right|
$$

количество ненулевых локальных линейных вероятностных соотношений в системе $\mathcal{L}$ для $r$ итераций XSLP-шифра.

Эта величина полностью определяется линейной средой, т. е. совокупностью линейных преобразований XSLP-шифра [1].

Определение 1 ([2]). Показателем рассеивания линейной среды XSLP-шифра с $r$ итерациями называется величина

$$
\theta_{\min }(r)=\min _{\mathcal{L} \in W} \theta_{\mathcal{L}}(r)
$$

Иногда подстановки (S-блоки), входящие в систему $\mathcal{L}$ с ненулевыми соотношениями, называют активными. Тогда $\theta_{\min }(r)$ представляет собой минимальное количество активных $S$-блоков. 
Используя показатель рассеивания линейной среды, можно получить, например, следующую оценку:

$$
\widetilde{\delta}_{\max }(r) \leqslant\left(\max _{i \in \overline{1, r}, j \in \overline{0, k^{2}-1}} \delta_{\pi_{i, j}}\right)^{\theta_{\min }(r)},
$$

где $\delta_{\pi_{i, j}}-$ наибольший модуль преобладания по всем ненулевым линейным вероятностным соотношениям (линейная характеристика) локальной подстановки $\pi_{i, j}$.

С учетом двойственности [1] показатель рассеивания линейной среды XSLP-шифра определяется для линейного и разностного методов одинаково, но сами значения, вообще говоря, могут быть различными.

Естественным образом возникают задачи оценки линейных (разностных) характеристик локальных подстановок и показателя рассеивания линейной среды. Настоящая работа посвящена второй задаче и связанным с ней вопросам.

Вычисление показателя рассеивания линейной среды основывается на рассеивающих характеристиках линейных преобразований $L_{i}$ и $\widehat{P}_{i}$.

Перестановки $m$-векторов в AES-подобных алгоритмах шифрования, а значит, и преобразования $\widehat{P}_{i}$ в XSLP-шифрах, обычно выбирают в соответствии со следующим определением.

Определение 2 ([2]). Будем говорить, что преобразование $\widehat{P}_{i}: V_{m k^{2}} \rightarrow V_{m k^{2}}$ является равномерно рассеивающим, если для соответствующей перестановки

$$
\begin{aligned}
& P_{i}=\left(\begin{array}{cccc}
0 & 1 & \cdots & k^{2}-1 \\
P_{i}(0) & P_{i}(1) & \cdots & P_{i}\left(k^{2}-1\right)
\end{array}\right)= \\
& =\left(\begin{array}{ccccccccc}
(0,0) & (1,0) & \cdots & (k-1,0) & \cdots & (0, k-1) & (1, k-1) & \cdots & (k-1, k-1) \\
P_{i}(0,0) & P_{i}(1,0) & \cdots & P_{i}(k-1,0) & \cdots & P_{i}(0, k-1) & P_{i}(1, k-1) & \cdots & P_{i}(k-1, k-1)
\end{array}\right)
\end{aligned}
$$

выполняется условие

$$
\left|\left\{\left[\frac{P_{i}\left(j_{0}, j_{1}\right)}{k}\right], j_{0} \in \overline{0, k-1}\right\}\right|=k \text { для всех } j_{1} \in \overline{0, k-1},
$$

т. е. $m$-векторы из каждого $m k$-вектора после перестановки распределяются равномерно по всем $m k$-векторам.

Для оценки рассеивающих свойств преобразований $L_{i}$ используют характеристики матриц $B_{i, j_{1}} \in \mathrm{GL}(m k, 2), j_{1}=0,1, \ldots, k-1$, из которых состоит это преобразование. Обозначим через $[x]$ количество ненулевых $m$-векторов в $m k$-векторе $x \in V_{m k}$. 
Определение 3 ([2]). Линейным коэффициентом рассеивания матрицы $M \in \mathrm{GL}(m k, 2)$ будем называть величину

$$
\rho_{L}(M)=\min _{l \in V_{m k} \backslash\{0\}}\left([l]+\left[l \cdot M^{T}\right]\right) .
$$

Определение 4 ([2]). Разностным коэффичиентом рассеивания матрицы $M \in \mathrm{GL}(m k, 2)$ будем называть величину

$$
\rho_{D}(M)=\min _{l \in V_{m k} \backslash\{0\}}([l]+[l \cdot M]) .
$$

Для любой матрицы $M \in \mathrm{GL}(m k, 2)$ справедливы неравенства $2 \leqslant \rho_{D}(M) \leqslant k+1$ и $2 \leqslant \rho_{L}(M) \leqslant k+1$. Известно, что верхние границы этих неравенств достигаются одновременно. В случае когда $\rho_{D}(M)=$ $=\rho_{L}(M)=k+1$, матрицу $M$ называют максимально рассеивающей.

Показатель рассеивания линейной среды XSLP-шифра полностью определяется перестановками $P_{i}, i=1,2, \ldots, r$, коэффициентами рассеивания матриц $B_{i, j_{1}}, \quad i=1,2, \ldots, r, \quad j_{1}=0,1, \ldots, k-1$, а также количеством итераций $r$. Таким образом, задача определения показателя рассеивания является оптимизационной задачей дискретной математики. С одной стороны, для ее решения существуют различные алгоритмические подходы.

1. Подход, основанный на переборе методом ветвей и границ [4].

2. Подход, основанный на решении систем линейных неравенств [11].

3. Подход, основанный на поиске с использованием графов [9].

С другой стороны, теоретический подход использовался авторами алгоритма AES при оценке показателя рассеивания четырех итераций этого шифра [8]. В [2] получена общая формула для показателя рассеивания XSLP-шифров с равномерно рассеивающими перестановками $\widehat{P}_{i}$ и максимально рассеивающими матрицами $B_{i, j_{1}}, i=1,2, \ldots, r, j_{1}=0,1, \ldots, k-1$.

\section{3. Графовая модель для XSLP-шифров с равномерно рассеивающими перестановками}

Для исследования рассеивающих свойств итерациями удобно использовать графовую модель XSLP-шифров с длиной блока $n=m k^{2}$ битов и $r$ итерациями [2].

Системе согласованных линейных (разностных) вероятностных соотношений $\mathcal{L}$ для XSLP-шифра поставим в соответствие ориентированный граф $c u$ стемы соотношений $\Gamma_{\mathcal{L}}$. Такой граф состоит из $r$ слоев вершин и $r+1$ слоев направленных ребер (дуг). Пусть далее $\mathcal{L}-$ система, например, линейных вероятностных соотношений. 
С некоторой вершиной $v_{i, j_{1}}$ слоя $i=1,2, \ldots, r$ ассоциируется матрица $B_{i, j_{1}}$ преобразования $L_{i}$, для которой в системе $\mathcal{L}$ присутствует ненулевое линейное вероятностное соотношение, задаваемое векторами-столбцами $t_{i, j_{1}}^{\prime}, t_{i, j_{1}}^{\prime \prime} \in V_{m k}^{*} \backslash\{0\}, j_{1}=0,1, \ldots, k-1$.

Укажем правило соединения вершин дугами. Рассмотрим соседние итерации с номерами $i$ и $i+1$, имеющие вид $X_{i} \cdot S_{i} \cdot L_{i} \cdot \widehat{P}_{i}$ и $X_{i+1} \cdot S_{i+1} \cdot L_{i+1} \cdot \widehat{P}_{i+1}$. Для преобразования $\widehat{P}_{i} \cdot X_{i+1} \cdot S_{i+1}$ линейное вероятностное соотношение задается векторами-столбцами $t_{i}^{\prime \prime}, t_{i+1}^{\prime} \in V_{m k^{2}}^{*}$, состоящими из $k^{2}$ векторов-столбцов $t_{i,\left(j_{0}^{\prime \prime}, j_{1}^{\prime \prime}\right)}^{\prime}, t_{i+1,\left(j_{0}^{\prime}, j_{1}^{\prime}\right)}^{\prime} \in V_{m}^{*}, j_{0}^{\prime}, j_{1}^{\prime}, j_{0}^{\prime \prime}, j_{1}^{\prime \prime} \in$ $\in \overline{0, k-1}$. По свойству преобразований $\widehat{P}_{i}, X_{i+1}, S_{i+1}$ каждому ненулевому вектору-столбцу $t_{i,\left(j_{0}^{\prime \prime}, j_{1}^{\prime \prime}\right)}$ соответствует вполне определенный ненулевой вектор-столбец $t_{i+1,\left(j_{0}^{\prime}, j_{1}^{\prime}\right)}^{\prime}$, где $\left(j_{0}^{\prime}, j_{1}^{\prime}\right)=P^{-1}\left(j_{0}^{\prime \prime}, j_{1}^{\prime \prime}\right)$. Тогда в графе $\Gamma_{\mathcal{L}}$ дуга проводится от вершины $v_{i, j_{1}^{\prime \prime}}$ к вершине $v_{i+1, j_{1}^{\prime}}$. В вершину $v_{1, j_{1}}$ слоя 1 графа $\Gamma_{\mathcal{L}}$ входят (не имея начала) дуги, соответствующие ненулевым векторамстолбцам $t_{1,\left(j_{0}, j_{1}\right)}^{\prime}, j_{0} \in \overline{0, k-1}$. Из вершины $v_{r, j_{1}}$ слоя $r$ графа $\Gamma_{\mathcal{L}}$ выходят (не имея окончания) дуги, соответствующие ненулевым векторам-столбцам $t_{r,\left(j_{0}, j_{1}\right)}^{\prime \prime}, j_{0} \in \overline{0, k-1}$.

Введем следующие обозначения, связанные с графом $\Gamma_{\mathcal{L}}$ :

$q_{i} \in \mathbb{N}-$ количество вершин на $i$-м слое $\Gamma_{\mathcal{L}}$,

$D_{i}=\left\{d_{i, 1}, d_{i, 2}, \ldots, d_{i, q_{i}}\right\}-$ набор степеней вершин $i$-го слоя $\Gamma_{\mathcal{L}}$,

$D_{i}^{\downarrow}=\left\{d_{i, 1}^{\downarrow}, d_{i, 2}^{\downarrow}, \ldots, d_{i, q_{i}}^{\downarrow}\right\}-$ набор степеней захода вершин $i$-го слоя $\Gamma_{\mathcal{L}}$,

$D_{i}^{\uparrow}=\left\{d_{i, 1}^{\uparrow}, d_{i, 2}^{\uparrow}, \ldots, d_{i, q_{i}}^{\uparrow}\right\}$ - набор степеней исхода вершин $i$-го слоя $\Gamma_{\mathcal{L}}$.

По определению $d_{i, j}=d_{i, j}^{\downarrow}+d_{i, j}^{\uparrow}$ для всех $i \in \overline{1, r}, j \in \overline{1, q_{i}}$. Из способа построения графа $\Gamma_{\mathcal{L}}$ следует, что

$$
\sum_{j=1}^{q_{i}}{d_{i, j}^{\uparrow}}_{i=1}^{q_{i+1}} \sum_{j=1}^{\downarrow}=: s_{i+1} \quad \text { для всех } \quad i \in \overline{1, r-1} .
$$

Определим также $s_{1}=\sum_{j=1}^{q_{1}} d_{1, j}^{\downarrow}$ и $s_{r+1}=\sum_{j=1}^{q_{1}} d_{r, j}^{\uparrow}$.

Условия согласованности системы $\mathcal{L} \in W$ для графа $\Gamma_{\mathcal{L}}$ означают, что необходимо выполнены условия

$$
\begin{aligned}
q_{i} \geqslant 1 \quad \text { для всех } \quad i \in \overline{1, r}, \\
d_{i, j}^{\downarrow} \geqslant 1, d_{i, j}^{\uparrow} \geqslant 1 \quad \text { для всех } \quad i \in \overline{1, r}, \quad j \in \overline{1, q_{i}} .
\end{aligned}
$$

Нетрудно заметить, что $\theta_{\mathcal{L}}(r)=\sum_{i=1}^{r} s_{i}=\sum_{i=1}^{r} \sum_{j=1}^{q_{i}} d_{i, j}^{\downarrow}$, поэтому дальнейшие исследования показателя рассеивания линейной среды XSLP-шифра и связанных с ним понятий можно проводить на языке теории графов. 
Далее рассматриваются XSLP-шифры с ограничениями на выбор составляющих преобразований. Пусть для всех $i \in \overline{1, r}$ преобразования $\widehat{P}_{i}$ являются равномерно рассеивающими. Пусть также для всех $i \in \overline{1, r}, j_{1} \in \overline{0, k-1}$ матрицы $B_{i, j_{1}} \in \mathrm{GL}(m k, 2)$ преобразований $L_{i} \in \mathrm{GL}\left(m k^{2}, 2\right)$ имеют линейный (разностный) коэффициент рассеивания $\rho$.

Заметим, что в [2] рассматривается случай $\rho=k+1$. Однако на практике встречается ситуация, когда $\rho<k+1$ (см., например, алгоритм Crypton [10]), исследование которой представляет отдельный интерес.

Введенные ограничения на рассматриваемый класс XSLP-шифров приводят к ограничениям на множество систем $\mathcal{L} \in W$, что выражается следующими условиями на графы $\Gamma_{\mathcal{L}}$ :

вершины $i$-го слоя соединяются с вершинами $(i+1)$-го слоя, $d_{i, j} \geqslant \rho$ для всех $i \in \overline{1, r}, \quad j \in \overline{1, q_{i}}, \quad$ где $\rho$ - коэффициент рассеивания матриц $B_{i, j}$.
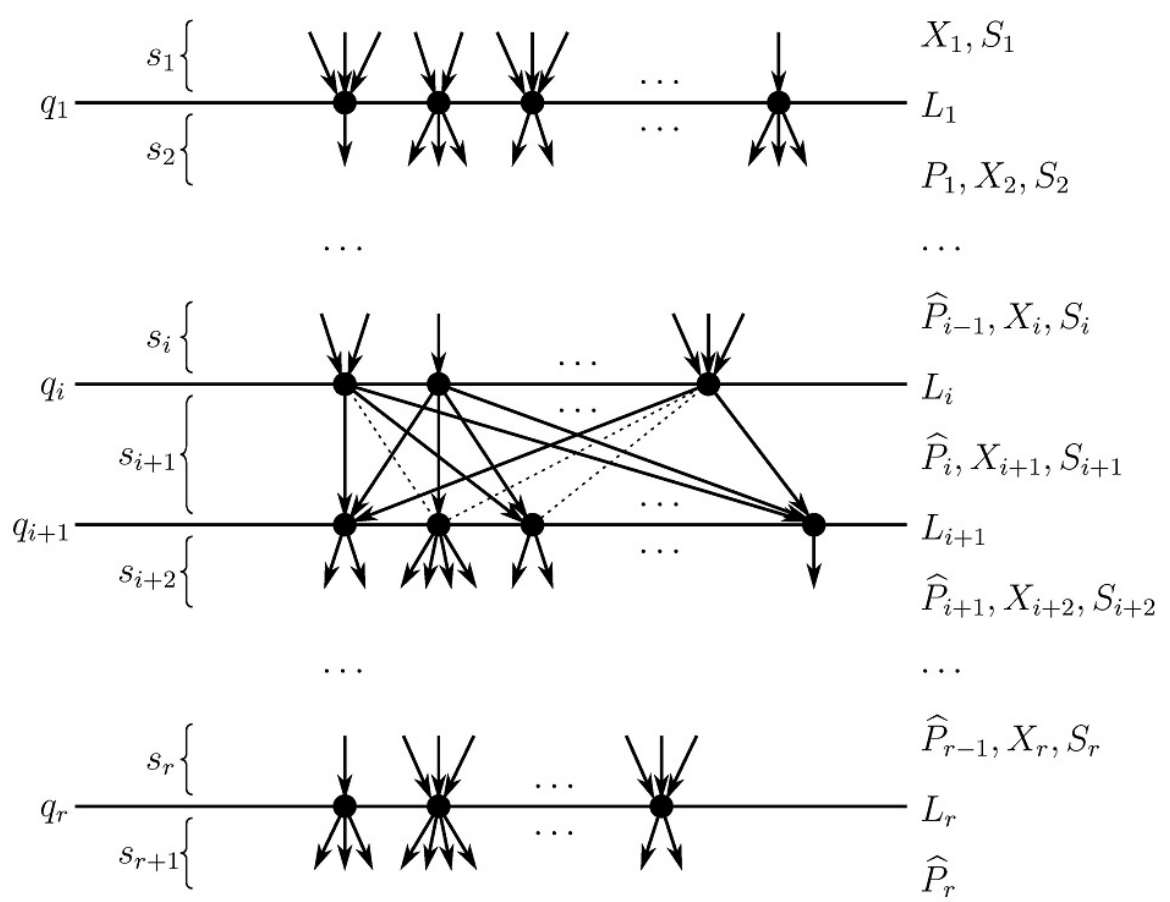

Рис. 3. Иллюстрация к определению графа $\Gamma_{\mathcal{L}}$ 
При $r \in \mathbb{N}$ множество всех $r$-слойных графов, удовлетворяющих условиям (12)-(15), обозначим $G(r)$. Отметим, что в графовой модели умышленно не вводятся ограничения сверху на количества вершин на каждом слое графа. При этом если для некоторого $\Gamma^{(r)} \in G(r)$ справедливы неравенства $q_{i} \leqslant k^{\prime}$, $d_{i, j}^{\downarrow} \leqslant k^{\prime}, d_{i, j}^{\uparrow} \leqslant k^{\prime}$ для всех $i \in \overline{1, r}, j \in \overline{1, q_{i}}$, то ему соответствует некоторая согласованная система соотношений для $r$ итераций XSLP-шифра с длиной блока $m k^{2}$ для всех $k \geqslant k^{\prime}$.

\section{4. Свойства $r$-слойных графов}

Для любого графа $\Gamma^{(r)} \in G(r)$ количество ребер (кроме выходящих из вершин $r$-го слоя) обозначим $S\left(\Gamma^{(r)}\right)=\sum_{i=1}^{r} s_{i}$. При каждом $r \in \mathbb{N}$ определена величина $S_{\min }(r)=\min _{\Gamma^{(r)} \in G(r)} S\left(\Gamma^{(r)}\right)$.

Определение 5. Будем называть граф $\Gamma^{(r)} \in G(r)$ реберно минимальным, если $S\left(\Gamma^{(r)}\right)=S_{\min }(r)$.

Теорема 1. Пусть $\Gamma^{(r)}$-реберно минимальный $r$-слойный граф. Тогда

$$
S\left(\Gamma^{(r)}\right)=S_{\min }(r)=\left\{\begin{array}{lll}
1, & \text { если } r=1, \\
\rho, & \text { если } r=2, \\
2 \rho-1, & \text { если } r=3, \\
\rho^{2}, & \text { если } r=4, \\
S_{\min }(r-4)+\rho^{2}, & \text { если } r>4 .
\end{array}\right.
$$

Доказательство. Применим метод математической индукции по $r$. База индукции включает случаи $r=1,2,3,4$. При $r=1$ и $r=2$ доказательство очевидно. Примеры реберно минимальных графов при данных значениях $r$ изображены на рис. 4.
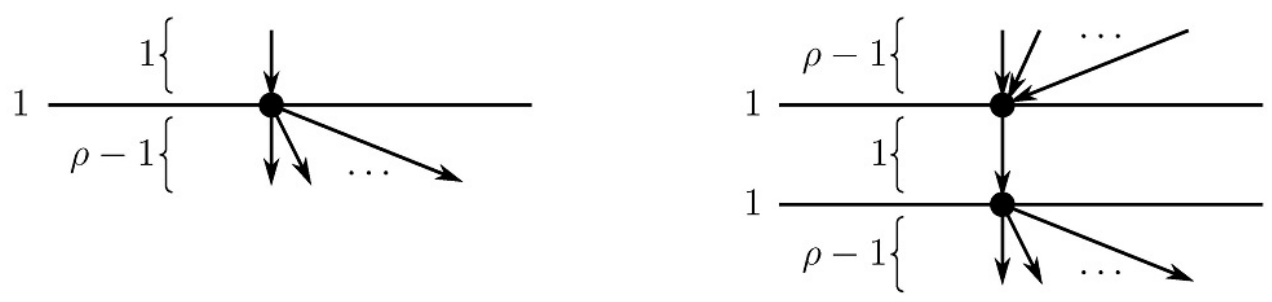

Рис. 4. Примеры 1- и 2-слойных графов 
Пусть $r=3$. Рассмотрим произвольный реберно минимальный 3 -слойный граф $\Gamma^{(3)}$. Заметим, что $S\left(\Gamma^{(3)}\right) \leqslant 2 \rho-1$, так как можно привести пример 3 -слойного графа с количеством ребер $2 \rho-1$ (рис. 5).

Докажем, что для графа $\Gamma^{(3)}$ всегда $q_{1}=1, q_{2}=1$. Действительно, учитывая указанную оценку, условие (15) и неравенства $s_{3} \geqslant q_{2} \geqslant 1$, $s_{1} \geqslant q_{1} \geqslant 1$, получаем:

$$
\begin{aligned}
& 2 \rho-1 \geqslant S\left(\Gamma^{(3)}\right)=\left(\sum_{j=1}^{q_{1}} d_{1, j}\right)+s_{3} \geqslant q_{1} \rho+1, \\
& 2 \rho-1 \geqslant S\left(\Gamma^{(3)}\right)=s_{1}+\sum_{j=1}^{q_{2}} d_{2, j} \geqslant q_{2} \rho+1,
\end{aligned}
$$

отсюда следует, что $q_{1} \leqslant 2-\frac{2}{\rho}$ и $q_{2} \leqslant 2-\frac{2}{\rho}$, поэтому $q_{1}=1, q_{2}=1$.

Теперь нетрудно заметить, что $s_{2}=1 \quad$ и $s_{1}=s_{3}=\rho-1$, значит, $S_{\min }(3)=S\left(\Gamma^{(3)}\right)=2 \rho-1$.
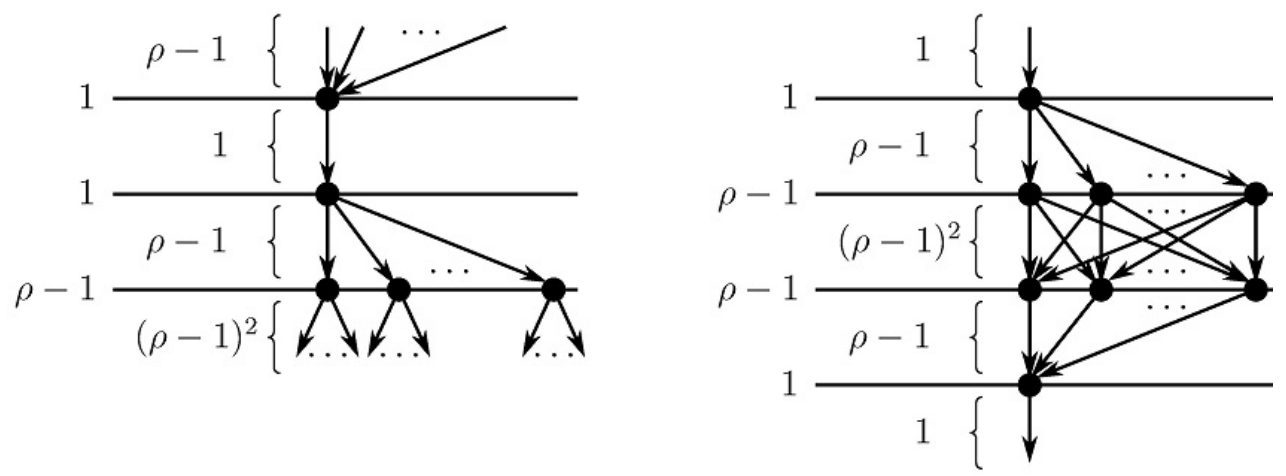

Рис. 5. Примеры 3- и 4-слойных графов

Пусть $r=4$. Рассмотрим произвольный реберно минимальный 4-слойный граф $\Gamma^{(4)}$. Заметим, что $S\left(\Gamma^{(4)}\right) \leqslant \rho^{2}$, так как можно привести пример 4 -слойного графа с количеством ребер $\rho^{2}$ (рис. 5).

Докажем, что для графа $\Gamma^{(4)}$ всегда $q_{1} \leqslant \rho-1, q_{2} \leqslant \rho-1, q_{3} \leqslant \rho-1$. Действительно,

$$
\rho^{2} \geqslant S\left(\Gamma^{(4)}\right)=\left(\sum_{j=1}^{q_{1}} d_{1, j}\right)+\sum_{j=1}^{q_{3}} d_{3, j} \geqslant\left(q_{1}+q_{3}\right) \rho,
$$

т. е. $q_{1}+q_{3} \leqslant \rho$. Поэтому с учетом (12) имеем $q_{1} \leqslant \rho-1$ и $q_{3} \leqslant \rho-1$. 
Далее,

$$
\rho^{2} \geqslant S\left(\Gamma^{(4)}\right)=s_{1}+\left(\sum_{j=1}^{q_{2}} d_{2, j}\right)+s_{4} \geqslant 1+q_{2} \rho+1>q_{2} \rho,
$$

значит, $q_{2} \leqslant \rho-1$.

При подсчете количества ребер можно считать, что в графе $\Gamma^{(4)}$ каждая вершина 1-го слоя соединена со всеми вершинами 2-го слоя. Действительно, если это не так и существуют не соединенные вершины $v_{1, j_{1}}, j_{1}=1,2, \ldots, q_{1}$, и $v_{2, j_{2}}, j_{2}=1,2, \ldots, q_{2}$, соответствующих слоев, то $d_{1, j_{1}}^{\uparrow} \leqslant q_{2}-1 \leqslant \rho-2$. Следовательно, $d_{1, j_{1}}^{\downarrow} \geqslant 2$, и можно соединить вершины $v_{1, j_{1}}$ и $v_{2, j_{2}}$ за счет исключения одного из ребер, входящих в $v_{1, j_{1}}$, оставив при этом $d_{1, j_{1}}^{\downarrow} \geqslant 1$. Аналогично, можно считать, что в графе $\Gamma^{(4)}$ каждая вершина 3-го слоя соединена со всеми вершинами 2-го слоя.

Если в графе $\Gamma^{(4)}$ в указанном смысле есть полная связность между слоями 1 и 2,2 и 3 , то

$$
\begin{aligned}
& d_{1, j}^{\downarrow} \geqslant \rho-d_{1, j}^{\uparrow}=\rho-q_{2} \quad \text { для всех } j \in \overline{1, q_{1}}, \\
& d_{2, j}^{\downarrow}=q_{1} \quad \text { для всех } \quad j \in \overline{1, q_{2}}, \\
& d_{3, j}^{\downarrow}=q_{2}, \quad d_{3, j}^{\uparrow} \geqslant \rho-d_{3, j}^{\uparrow}=\rho-q_{2} \quad \text { для всех } \quad j \in \overline{1, q_{3}}, \\
& q_{3} \geqslant \rho-d_{2, j}^{\downarrow}=\rho-q_{1} \quad \text { для всех } j \in \overline{1, q_{2}} .
\end{aligned}
$$

Воспользуемся формулами (17) для оценки количества ребер в графе $\Gamma^{(4)}$ :

$$
\begin{aligned}
S\left(\Gamma^{(4)}\right) & =\sum_{j=1}^{q_{1}} d_{1, j}^{\downarrow}+\sum_{j=1}^{q_{2}} d_{2, j}^{\downarrow}+\sum_{j=1}^{q_{3}} d_{3, j}^{\downarrow}+\sum_{j=1}^{q_{3}} d_{3, j}^{\uparrow} \geqslant \\
& \geqslant q_{1}\left(\rho-q_{2}\right)+q_{2} q_{1}+q_{3} q_{2}+q_{3}\left(\rho-q_{2}\right)=\left(q_{1}+q_{3}\right) \rho \geqslant \rho^{2} .
\end{aligned}
$$

Так как последняя оценка достижима, то $S_{\min }(4)=S\left(\Gamma^{(4)}\right)=\rho^{2}$.

Покажем, что для любого $r>4$ из справедливости (16) при $r^{\prime}<r$ следует справедливость (16) при $r^{\prime}=r$. Заметим сначала, что $S_{\min }(r)$ не может превосходить значения, задаваемого формулами (16), так как это значение достижимо. Для построения соответствующего графа необходимо к объединению из $\left[\frac{r}{4}\right]$ реберно минимальных 4-слойных графов добавить реберно минимальный $\left\{\frac{r}{4}\right\}$-слойный граф (если 4 не делит $r$ ), где $\left[\frac{r}{4}\right]-$ целая часть числа $\frac{r}{4},\left\{\frac{r}{4}\right\}$ - остаток от деления $r$ на 4 . 
Принцип построения таких графов легко понять по рис. 6.

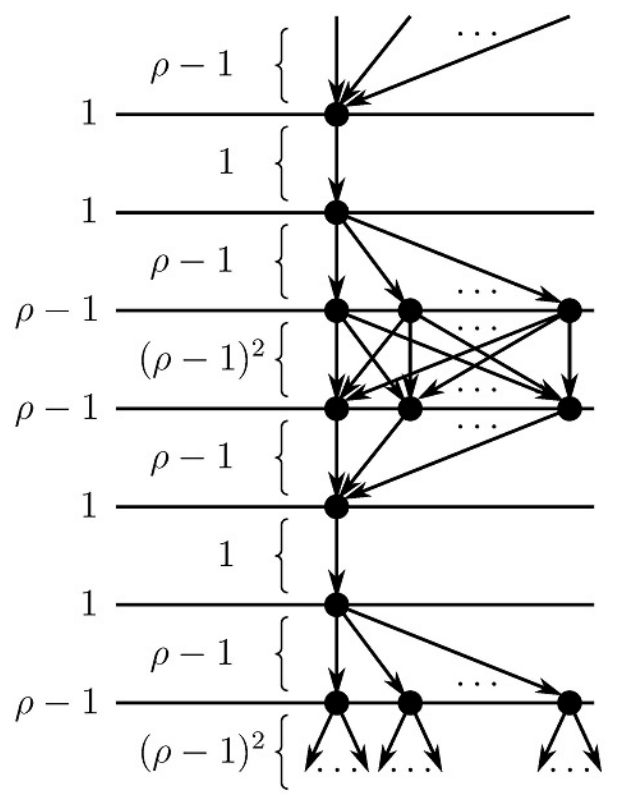

Рис. 6. Пример 7-слойного графа

Рассмотрим произвольный $r$-слойный граф $\Gamma^{(r)}$. Представим его в виде двух графов: $\Gamma^{(4)}-$ четыре верхних слоя $\Gamma^{(r)}$ и $\Gamma^{(r-4)}-(r-4)$ нижних слоев $\Gamma^{(r)}$. При этом оба графа $\Gamma_{1}^{(4)}$ и $\Gamma_{2}^{(r-4)}$ наследуют $s_{5}$ ребер между четвертым и пятым слоями вершин $\Gamma^{(r)}$. По предположению индукции для числа ребер графа $\Gamma^{(r)}$ имеем:

$$
S\left(\Gamma^{(r)}\right)=S\left(\Gamma_{1}^{(4)}\right)+S\left(\Gamma_{2}^{(r-4)}\right) \geqslant S_{\min }(4)+S_{\min }(r-4)=\rho^{2}+S_{\min }(r-4) .
$$

Доказываемая формула следует из достижимости этой оценки.

Согласно доказательству теоремы 1 минимально возможное количество ребер достигается по крайней мере на одном $r$-слойном графе, для которого $q_{i} \leqslant k^{\prime}, \quad d_{i, j}^{\downarrow} \leqslant k^{\prime}, \quad d_{i, j}^{\uparrow} \leqslant k^{\prime}$ при всех $i \in \overline{1, r}, \quad j \in \overline{1, q_{i}}$. Поэтому можно утверждать, что $S_{\min }(r)=\theta_{\min }(r)$, и для вычисления показателя рассеивания линейной среды XSLP-шифров можно использовать формулу (16).

В некоторых криптографических задачах, в частности при оценке стойкости SP-сетей с локальными подстановками из $S\left(V_{m k}\right)$, может оказаться существенным не только количество ребер, но и количество вершин в соответствующих графах. Для любого $\Gamma^{(r)} \in G(r)$ количество его вершин обозначим $Q\left(\Gamma^{(r)}\right)=\sum_{i=1}^{r} q_{i}$. Положим $Q_{\min }(r)=\min _{\Gamma^{(r)} \in G(r)} Q\left(\Gamma^{(r)}\right)$. 
Определение 6. Будем называть $r$-слойный граф $\Gamma^{(r)} \in G(r)$ вериинно минимальным, если $Q\left(\Gamma^{(r)}\right)=Q_{\min }(r)$.

Теорема 2. Пусть $\Gamma^{(r)} \in G(r)$ - вериинно минимальный $r$-слойный граф. Тогда

$$
Q\left(\Gamma^{(r)}\right)=Q_{\min }(r)=\left\{\begin{array}{lll}
1, & \text { если } r=1, \\
2, & \text { если } r=2, \\
\rho+1, & \text { если } r=3, \\
2 \rho, & \text { если } r=4, \\
Q_{\min }(r-4)+2 \rho, & \text { если } r>4 .
\end{array}\right.
$$

Доказательство. Воспользуемся идеей доказательства теоремы 1 и применим метод математической индукции по $r$. При $r=1$ и $r=2$ доказательство очевидно. Вершинно минимальными графами при данных значениях $r$ являются, например, реберно минимальные графы, представленные на рис. 4.

Рассмотрим вершинно минимальный 3 -слойный граф $\Gamma^{(3)}$. С одной стороны, $Q\left(\Gamma^{(3)}\right) \leqslant \rho+1$, так как можно привести пример 3-слойного графа с количеством вершин $\rho+1$ (рис. 5). С другой стороны, так как $q_{2} \geqslant 1$ и $d_{2, j} \geqslant \rho$ для всех $j \in \overline{1, q_{2}}$, то $q_{1}+q_{3} \geqslant \rho$. Поэтому $Q\left(\Gamma^{(3)}\right) \geqslant \rho+1$.

Рассмотрим вершинно минимальный 4 -слойный граф $\Gamma^{(4)}$. Легко заметить, что $Q\left(\Gamma^{(4)}\right)=q_{1}+q_{2}+q_{3}+q_{4} \leqslant 2 \rho$, так как можно привести пример 4 -слойного графа с количеством вершин $2 \rho$ (рис. 5). Вместе с тем $q_{1}+q_{3} \geqslant \rho$ и $q_{2}+q_{4} \geqslant \rho$ ввиду (12) и (15). Поэтому $q_{1}+q_{2}+q_{3}+q_{4} \geqslant 2 \rho$. Из этих двух неравенств следует искомое равенство.

Покажем, что для любого $r>4$ из справедливости (18) при $r^{\prime}<r$ следует справедливость (18) при $r^{\prime}=r$. Заметим, что $Q_{\min }(r)$ не может превосходить значение, задаваемое формулами (18), так как это значение достижимо, например, для реберно минимальных $r$-слойных графов из доказательства теоремы 1.

Рассмотрим произвольный $r$-слойный граф $\Gamma^{(r)}$. Разобьем его на два графа: $\Gamma^{(4)}-$ четыре верхних слоя $\Gamma^{(r)}$ и $\Gamma^{(r-4)}-(r-4)$ нижних слоев $\Gamma^{(r)}$. По предположению индукции для числа вершин графа $\Gamma^{(r)}$ :

$$
Q\left(\Gamma^{(r)}\right)=Q\left(\Gamma_{1}^{(4)}\right)+Q\left(\Gamma_{2}^{(r-4)}\right) \geqslant Q_{\min }(4)+Q_{\min }(r-4)=2 \rho+Q_{\min }(r-4) .
$$

Доказываемая формула следует из достижимости этой оценки.

Автор работы благодарен Ф. М. Малышеву за помощь в подготовке статьи и ценные замечания к первому ее варианту. 


\section{Список литературы}

[1] Малышев Ф. М., “Двойственность разностного и линейного методов в криптографии”, Математические вопросы криптографии, 5:3 (2014), 35-47.

[2] Малышев Ф. М., Трифонов Д. И, “Рассеивающие свойства XSLP-шифров”, Математические вопросы криптографии, 7:3 (2016), 47-60.

[3] Barreto P. S. L. M., Rijmen V., “The Anubis Block Cipher”, Submission to NESSIE (2000).

[4] Biryukov A., Nikolic I., "Automatic search for related-key differential characteristics in byteoriented block ciphers: applications to AES, Camellia, Khazad and others", Lect. Notes Comput. Sci., 6110, 2010, 322-344.

[5] Borst J., "The Block Cipher: GrandCru”, Submission to NESSIE (2000).

[6] Daemen J., Knudsen L., Rijmen V., “The Block Cipher Square”, Lect. Notes Comput. Sci., 1267, 1997, 149-165.

[7] Daemen J., Rijmen V., “The Block Cipher BKSQ”, Lect. Notes Comput. Sci., 1820, 2000, 236245.

[8] Daemen J., Rijmen V., The Design of Rijndael: AES - The Advanced Encryption Standard, Heidelberg etc. : Springer, 2002, 238 p.

[9] Fouque P.-A., Jean J., Peyrin T., "Structural evaluation of AES and chosen-key distinguisher of 9-round AES-128”, Lect. Notes Comput. Sci., 8042, 2013, 183-203.

[10] Lim C., "A revised version of Crypton: Crypton v1.0”, Lect. Notes Comput. Sci., 1636, 1999, $31-45$.

[11] Mouha N., Wang Q., GuD., Preneel B., "Differential and linear cryptanalysis using mixedinteger linear programming”, Lect. Notes Comput. Sci., 7537, 2012, 57-76. 